\title{
Polymerization of Methyl and Ethyl Methacrylates Initiated with Alkali-Metal Alkoxide Derivatives of Poly(ethylene oxide)
}

\author{
Masao Tomol, Yoshio Shibayama, and Hiroshi Kakiuchi \\ Department of Applied Chemistry, Faculty of Engineering, Yokohama \\ National University, Minami-ku, Yokohama 233, Japan.
}

(Received September 2, 1975)

\begin{abstract}
The polymerization of methacrylates with alkali-metal alkoxide derivatives of poly(ethylene oxide) (PEO) yielded copolymers which consisted of PEO and polymethacrylates containing block and graft units. The block copolymerization was initiated by addition of the alkoxide to the carbon-carbon double bond of methacrylates, and the graft reaction onto the block copolymer formed was thought to proceed by transesterification between the pendant ester groups of the copolymer and the unreacted alkoxide. The grafting of the alkoxide derivative of PEO having two terminal hydroxyl groups resulted in formation of cross-linked copolymers, while the polymerization with the alkoxide having one active end gave soluble copolymers. The relative amount of the grafted ester groups in the copolymers was estimated by NMR spectroscopy.

KEY WORDS Anionic Polymerization / Block Copolymer / Graft Copolymer / Poly(ethylene oxide) / Alkyl Methacrylate / Alkali-Metal Alkoxide / Crosslinking / Transesterification /
\end{abstract}

In a previous paper, ${ }^{1}$ it was shown that the polymerization of methyl methacrylate (MMA) with alkali-metal salts of 2 -substituted ethanols such as 2-methoxyethanol and 2-dimethylaminoethanol proceeded easily in the presence of hexamethylphosphoramide (HMPA). In this paper, the polymerization of MMA and ethyl methacrylate (EMA) with alkali-metal alkoxide derivatives of poly(ethylene oxide) (PEO) has been investigated, since the formation of block copolymers, poly(ethylene oxide- $b$-methacrylates), was expected from the similarity of structure between 2-methoxyethanol and the PEO carrying terminal hydroxyl groups. The block or graft copolymers composed of polymers having different properties, e.g., rubbery and glassy or hydrophilic and hydrophobic, have been noted as a kind of new prospective polymer materials. ${ }^{2}$ PEO has hydrophilic and rather rubbery properties, while polymethacrylates have hydrophobic and glassy ones. For example, poly(methyl methacrylate- $g$-ethylene oxide) have already been synthesized by the grafting of alkoxide derivatives of PEO onto poly(methyl methacrylate). ${ }^{3}$

In this study, the synthesis of poly(ethylene oxide- $b$-methacrylates) was attempted by means of the anionic polymerization of vinyl monomers
(MMA and EMA) with macromolecular initiators (alkoxide derivatives of PEO). Such block copolymers, which consist of alkylene oxide and vinyl or diene monomers, have already been synthesized by radical copolymerization, ${ }^{4}$ but not by anionic polymerization.

\section{EXPERIMENTAL}

\section{Reagents}

Tetrahydrofuran (THF) was distilled over metallic sodium under a nitrogen atmosphere. HMPA was refluxed and distilled over calcium hydride in vacuo. PEO having terminal hydroxyl groups was dried by azeotropic 'distillation in toluene. $^{3}$ 2-Methoxyethanol was refluxed and distilled over calcium hydride under a nitrogen atmosphere. Ethylene oxide (Nippon Shokubai Kagaku) was used without further purification. MMA and EMA were purified by conventional methods, refluxed, and distilled over calcium hydride under vacuum just before use. Sodium and potassium alkoxide derivatives of PEO or 2-methoxyethanol were prepared by the metallation of the alcohols with the corresponding alkali metal-naphthalene complexes in THF under a nitrogen atmosphere. ${ }^{3}$ The concentra- 
tion of the alkoxides was predetermined by acidbase titration.

\section{Polymerization Procedure}

The polymerization was carried out by the following three methods under a nitrogen atmosphere:

Polymerization of MMA with Alkali-Metal Salts of PEO Having Two Terminal Hydroxyl Groups. In a $30-\mathrm{m} l$ polymerization tube MMA and solvents were charged, and the polymerization was initiated by introducing a THF solution of the alkoxides derived from PEO. When the alkoxide solution was highly viscous, MMA was finally introduced to the solution. After a given time the polymerization mixture was poured into methanol. The precipitated polymer was collected by filtration, washed with methanol, and dried in vacuo at room temperature. The polymer obtained was concluded to be a copolymer of MMA and PEO on the basis of the following facts: (1) the homopolymerization of MMA with alkali-metal methoxides, which might be formed by transfer or graft reactions in the polymerization of MMA with the alkoxides derived from PEO, did not occur under the conditions similar to those of the polymerization with the alkoxides derived from PEO; (2) the unreacted PEO was soluble in methanol. When the polymer was a mixture of soluble and insoluble copolymers, the mixture was extracted with benzene at the reflux temperature. The insoluble part was separated by filtration or centrifugation, washed with benzene, and dried in vacuo. The filtrate containing the soluble copolymer was concentrated, poured into methanol, filtrated, and dried.
Polymerization of MMA and EMA with Living Polymers of Ethylene Oxide. In a $30-\mathrm{ml}$ polymerization tube equipped with a self-sealing rubber cap, ethylene oxide and HMPA were charged and the mixture was brought to the polymerization temperature. The polymerization of ethylene oxide was initiated by introducing a THF solution of 2-methoxyethoxides with a syringe through the rubber cap. After a given time interval MMA or EMA were introduced into the solution of living poly(ethylene oxide).

Polymerization of EMA with Alkali-Metal Salts of PEO Having One Terminal Hydroxyl Group. PEO having one terminal hydroxyl group was obtained by quenching living poly(ethylene oxide) produced by use of 2-methoxyethoxides as an initiator. The PEO was reconverted to alkalimetal salts by metallation in THF. The polymerization of EMA was initiated by introducing EMA into a solution of this alkoxide.

\section{Measurements}

Reduced viscosities were measured in a benzene solution at $25^{\circ} \mathrm{C}$. The NMR spectra of the copolymer solution in chlorobenzene or nitromethane were recorded at $120^{\circ} \mathrm{C}$ on a JNM C$60 \mathrm{H}$ spectrometer.

\section{RESULTS}

Polymerization of MMA with Alkali-Metal Alkoxide Derivatives of PEO Having Two Terminal Hydroxyl Groups

The syntheses of triblock copolymers of PEO and MMA were attempted by the following scheme.

$$
\begin{gathered}
\left.\left.\mathrm{HO}+\mathrm{CH}_{2} \mathrm{CH}_{2} \mathrm{O}\right)_{-}-\mathrm{H} \frac{\mathrm{Na}^{+} \mathrm{C}_{10} \mathrm{H}_{10}-}{\mathrm{THF}} \rightarrow \mathrm{Na}^{+-} \mathrm{O}+\mathrm{CH}_{2} \mathrm{CH}_{2} \mathrm{O}\right)_{\overline{n-1}} \mathrm{CH}_{2} \mathrm{CH}_{2} \mathrm{O}^{-} \mathrm{Na}^{+} \\
\frac{\text { MMA }}{\mathrm{THF}(+\mathrm{HMPA})} \rightarrow\left(\mathrm{MMA}-\frac{-}{x} \mathrm{O}+\mathrm{CH}_{2} \mathrm{CH}_{2} \mathrm{O}-_{\bar{n}}-(-\mathrm{MMA})_{y}\right.
\end{gathered}
$$

The results are shown in Table I. The polymerization of MMA with sodium salt of PEO (molecular weight, $M_{n}, 200$ ) proceeded readily both in THF and in a THF-HMPA mixture. The polymerization with sodium salt of PEO $\left(M_{n} 1000\right)$ did not proceed in THF, but proceeded in the presence of large amounts of HMPA. On the other hand, the corresponding potassium salt could polymerize MMA even in the absence of HMPA. The polymerization with bifunctional alkali salts derived from PEO $\left(M_{n}\right.$ 1000) gave both benzene-soluble and -insoluble copolymers. The polymerization with alkali-metal alkoxide derivatives, in which $21-49 \%$ of the terminal hydroxyl groups of PEO $\left(M_{n} 2000\right.$ and $6000)$ were metallated, gave also soluble and 
M. Tomoi, Y. Shibayama, and H. KakiUchi

Table I. Polymerization of MMA with alkali metal alkoxide derived from PEO ${ }^{a}$

\begin{tabular}{|c|c|c|c|c|c|c|c|c|}
\hline \multirow{2}{*}{ No. } & \multirow{2}{*}{$\underset{\mathrm{mol}}{\mathrm{PEO} \times 10^{4}}$} & \multirow{2}{*}{$\left(M_{n}\right)$} & \multirow{2}{*}{$\begin{array}{l}\text { Metallated } \\
\mathrm{OH} \text { group, }\end{array}$} & \multirow{2}{*}{$\underset{\text { ion }}{\text { Counter }}$} & \multirow{2}{*}{$\begin{array}{l}\text { HMPA, } \\
\mathrm{m} l\end{array}$} & \multicolumn{3}{|c|}{ Yield, \% } \\
\hline & & & & & & Soluble & (Total) & Insoluble \\
\hline 1 & 6.55 & $(200)$ & 100 & $\mathrm{Na}^{+}$ & 0 & 32 & (32) & 0 \\
\hline 2 & 5.50 & $(200)$ & 100 & $\mathrm{Na}^{+}$ & 1 & 42 & (42) & 0 \\
\hline 3 & 4.60 & $(1000)$ & 100 & $\mathrm{Na}^{+}$ & 0 & & $(0)$ & \\
\hline 4 & 3.55 & $(1000)$ & 100 & $\mathrm{Na}^{+}$ & 1 & & (0) & \\
\hline 5 & 4.60 & $(1000)$ & 100 & $\mathrm{Na}^{+}$ & 5 & & (8) & \\
\hline 6 & 4.60 & $(1000)$ & 100 & $\mathrm{Na}^{+}$ & 10 & 24 & (37) & 13 \\
\hline 7 & 3.30 & $(1000)$ & 100 & $\mathbf{K}^{+}$ & 0 & & (41) & \\
\hline 8 & 3.30 & $(1000)$ & 100 & $\mathbf{K}^{+}$ & 1 & 30 & (40) & 10 \\
\hline 9 & 3.30 & $(1000)$ & 100 & $\mathbf{K}^{+}$ & 5 & & (46) & \\
\hline 10 & 11.2 & $(2000)$ & 49 & $\mathrm{Na}^{+}$ & 0 & & $(0)$ & \\
\hline 11 & 11.2 & $(2000)$ & 49 & $\mathrm{Na}^{+}$ & 5 & & (58) & \\
\hline 12 & 11.2 & $(2000)$ & 21 & $\mathrm{Na}^{+}$ & 10 & $22^{b}$ & (34) & 12 \\
\hline 13 & 19.3 & $(2000)$ & 49 & $\mathrm{~K}^{+}$ & 0 & $15^{b}$ & (18) & 3 \\
\hline 14 & 9.00 & $(2000)$ & 25 & $\mathrm{~K}^{+}$ & 10 & $6^{b}$ & $(8)$ & 25 \\
\hline 15 & 9.50 & $(6000)$ & 32 & $\mathrm{~K}^{+}$ & 5 & & (32) & \\
\hline 16 & 11.2 & $(6000)$ & 39 & $\mathrm{~K}^{+}$ & 5 & 6 & (22) & 16 \\
\hline 17 & 8.70 & $(6000)$ & 23 & $\mathbf{K}^{+}$ & 0 & 1 & (9) & 8 \\
\hline
\end{tabular}

a Solvent, THF + HMPA, $20 \mathrm{ml}$; MMA, $1.87 \times 10^{-2} \mathrm{~mol}$ (No. $16,3.74 \times 10^{-2} \mathrm{~mol}$ ); temp, room temperature; time, $15 \mathrm{hr}$.

b Copolymer compositions, [MMA]:[EO], and $\eta_{\mathrm{sp}} / c(\mathrm{~d} l / \mathrm{g})$ of soluble parts were as follows: No. 12, $3: 1,0.47$; No. $13,13: 1,0.52$; No. $14,4: 1,0.40$.

insoluble copolymers. The yield of the insoluble copolymer increased in the case of the polymerization with potassium salts and of the polymerization in the presence of large amounts of HMPA.

Polymerization of MMA and EMA with Living Poly(ethylene oxide)
The anionic polymerizations of ethylene oxide in polar solvents such as HMPA $^{5}$ and dimethylsulfoxide ${ }^{6}$ have been confirmed to cause a living polymerization. The syntheses of block copolymers of ethylene oxide and MMA were attempted by the reaction of living PEO with MMA as follows.

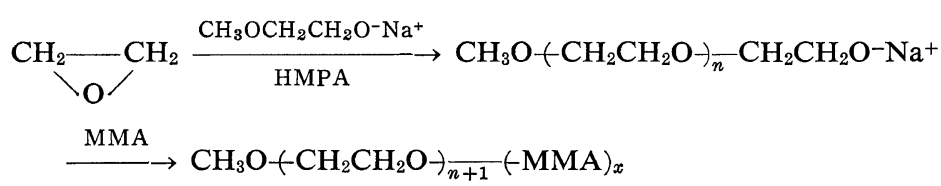

The results are shown in Table II. The insoluble copolymer was not formed in the case of using an initiator in which the polymer alkoxide carried one living end per chain. The tacticity of the poly(methyl methacrylate) part in the copolymer was measured by NMR spectroscopy: ${ }^{7}$ it was predominantly syndiotactic and heterotactic, as had been expected from the polymerization results obtained in the presence of HMPA. ${ }^{1}$

It was not clear whether or not there was a graft reaction of the alkoxide in this case, for
PEO (- $\mathrm{OCH}_{2} \mathrm{CH}_{2} \mathrm{O}-$ ) and poly(methyl methacrylate) $\left(-\mathrm{OCH}_{3}\right)$ gave an overlapped resonance peak at $\delta=\sim 3.6$. In order to make clear the possible occurrence of the graft reaction, the polymerization of EMA with living PEO was carried out: the methylene protons of PEO (- $\mathrm{OCH}_{2} \mathrm{CH}_{2} \mathrm{O}$-) and poly(ethyl methacrylate) $\left(-\mathrm{OCH}_{2} \mathrm{CH}_{3}\right)$ can be distinguished by means of NMR spectroscopy. Figure 1 shows the NMR spectrum of the copolymer. The structure of the copolymer could be represented as follows. 
Table II. Polymerization of MMA and EMA with living poly(ethylene oxide) ${ }^{\mathrm{a}}$

\begin{tabular}{|c|c|c|c|c|c|c|c|c|c|c|}
\hline \multirow{2}{*}{ No. } & \multirow{2}{*}{$\begin{array}{c}\text { Ethylene } \\
\text { oxide } \\
\times 10^{2} \\
\text { mol }\end{array}$} & \multirow{2}{*}{$\begin{array}{c}\text { Initiator } \\
\times 10^{4}, \mathrm{~b} \\
\text { mol }\end{array}$} & \multirow{2}{*}{$\begin{array}{c}\text { Monomer } \\
\times 10^{2} \\
\text { mol }\end{array}$} & \multirow{2}{*}{$\begin{array}{c}\text { HMPA, } \\
\mathrm{m} l\end{array}$} & \multirow{2}{*}{$\underset{\%}{\text { Yield, }}$} & \multirow{2}{*}{$\begin{array}{c}\text { Copolymer } \\
\text { composition, } \\
\text { [MMA or } \\
\text { EMA]/[EO] }\end{array}$} & \multirow{2}{*}{$\begin{array}{c}\eta_{\mathrm{sp}} / c,{ }^{\mathrm{c}} \\
\mathrm{d} l / \mathrm{g}\end{array}$} & \multicolumn{3}{|c|}{ Tacticity, $\%$} \\
\hline & & & & & & & & $I$ & $H$ & $S$ \\
\hline 18 & 4.10 & 0.80 & MMA 3.74 & 5 & 7 & 2.1 & 0.58 & 6 & 34 & 60 \\
\hline 19 & 13.1 & 3.00 & MMA 3.74 & 10 & 53 & 0.3 & 0.42 & 10 & 45 & 45 \\
\hline 20 & 5.60 & 3.80 & MMA 9.35 & 5 & 32 & 8.0 & 0.26 & 13 & 47 & 40 \\
\hline 21 & 7.40 & 5.70 & MMA 3.74 & 5 & 46 & 1.2 & 0.19 & 14 & 42 & 44 \\
\hline 22 & 11.0 & 5.70 & EMA 8.00 & 10 & 29 & $2.3^{d}$ & 0.22 & - & - & - \\
\hline 23 & 1.70 & $1.10^{\mathrm{e}}$ & EMA 2.40 & 1 & 13 & $14^{\mathrm{d}}$ & 0.25 & - & - & - \\
\hline
\end{tabular}

a Solvent, THF +HMPA, $10 \mathrm{ml}$; temp, room temperature; time (polymerization of ethylene oxide), $72 \mathrm{hr}$; time (polymerization of methacrylate), $24 \mathrm{hr}$.

b Sodium 2-methoxyethoxide.

c Benzene solution at $25^{\circ} \mathrm{C}, c=0.25 \mathrm{~g} / \mathrm{d} l$.

d The ratio of grafted esters to total esters was as follows: No. 22, 4.1\%; No. 23, 6.2\%.

- Potassium 2-methoxethoxide.<smiles>CCOC(=O)C(C)(C)CC(=O)C(C)(C)COCCOC</smiles>

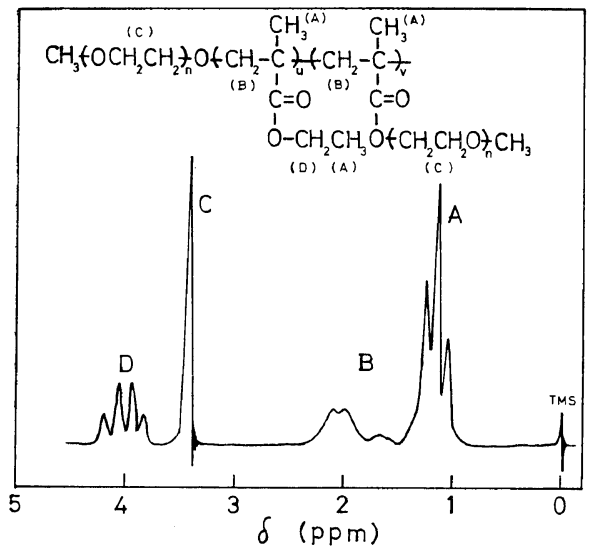

Figure 1. NMR spectrum of PEO-EMA copolymer (No. 22 in Table II): chlorobenzene solution, $120^{\circ} \mathrm{C}$.

Assignments of signals could be made as shown in the figure. The ratio of the numbers of grafted ester groups to all of the ester groups, $v /(u+v)$, was calculated from the relative intensity between the methylene protons of the pendant esters and of the backbone. The ratio was calculated as follows

$$
\frac{v}{u+v}=1-\frac{u}{u+v}=1-\frac{D_{\mathrm{CH}_{2}}}{B_{\mathrm{CH}_{2}}}
$$

where $B_{\mathrm{CH}_{2}}$ and $D_{\mathrm{CH}_{2}}$ are the resonance areas of methylene protons $\mathrm{CH}_{2}(\mathrm{~B})$ and $\mathrm{CH}_{2}(\mathrm{D})$ in Figure 1, respectively. The percentage of the grafted ester groups thus estimated in the PEOEMA copolymer was 4-6\%.

The polymerization of EMA was also carried out in the absence of HMPA. In this case, PEO having one terminal hydroxyl group was prepared by the polymerization of ethylene oxide with potassium 2-methoxyethoxide, and was metallated in THF. The results of the polymerization are shown in Table III.

$$
\begin{aligned}
& \mathrm{CH}_{3}+\mathrm{OCH}_{2} \mathrm{CH}_{2}-\frac{-}{n} \mathrm{OH} \\
& \frac{\mathrm{K}^{+} \mathrm{C}_{10} \mathrm{H}_{10}{ }^{-}}{\mathrm{THF}} \rightarrow \mathrm{CH}_{3}\left(\mathrm{OCH}_{2} \mathrm{CH}_{2}\right)_{n}-\mathrm{O}^{-} \mathrm{K}^{+} \\
& \left.\stackrel{\mathrm{EMA}}{\rightarrow} \mathrm{CH}_{3}+\mathrm{OCH}_{2} \mathrm{CH}_{2}+{ }_{n} \mathrm{O}+\mathrm{EMA}\right)_{x}
\end{aligned}
$$

In this case also, the percentage of the grafted ester groups was ca. $5 \%$, i.e., the graft copolymer containing one PEO side chain per about 20 EMA units was obtained.

The copolymers having high molecular weights 
Table III. Polymerization of EMA with potassium salts of PEO having one terminal hydroxyl group ${ }^{\mathrm{a}}$

\begin{tabular}{ccccccccc}
\hline No. & PEO, g & $\begin{array}{c}\eta_{\mathrm{sp}} / c, \mathrm{~b} \\
\mathrm{~d} l / \mathrm{g}\end{array}$ & $\begin{array}{c}\text { EMA } \\
\times 10^{2}, \\
\mathrm{~mol}\end{array}$ & $\begin{array}{c}\text { Feed ratio, } \\
{[\mathrm{EMA}] /[\mathrm{EO}]}\end{array}$ & $\begin{array}{c}\text { Yield, } \\
\%\end{array}$ & $\begin{array}{c}\text { Copolymer } \\
\text { composition, } \\
{[\text { EMA]/[EO] }}\end{array}$ & $\begin{array}{c}\eta_{\mathrm{sp}} / \boldsymbol{c}, \mathrm{b} \\
\mathrm{d} l / \mathrm{g}\end{array}$ & $\begin{array}{c}\text { Grafted ester, } \\
\%\end{array}$ \\
\hline 24 & 1.90 & $(0.07)$ & 4.80 & 1.11 & 22 & 21.5 & 0.31 & $5.7(5.5)$ \\
25 & 2.20 & $(0.08)$ & 2.40 & 0.48 & 10 & 10.0 & - & $5.0(5.0)$ \\
26 & 1.40 & $(0.08)^{\mathrm{d}}$ & 4.80 & 1.51 & 34 & 11.3 & 0.53 & $6.7(3.1)$ \\
27 & 0.90 & $(0.09)$ & 4.80 & 2.34 & 50 & 17.5 & 0.30 & $2.9(0.5)$ \\
28 & 1.40 & $(0.09)$ & 6.40 & 2.01 & 40 & 14.5 & 0.38 & $3.6(6.7)$ \\
\hline
\end{tabular}

a Solvent, THF, $10 \mathrm{ml}$; temp, $0^{\circ} \mathrm{C}$; time, $24 \mathrm{hr}$.

b Benzene solution at $25^{\circ} \mathrm{C}, c=0.25 \mathrm{~g} / \mathrm{d} l$.

c The values were determined from the ratio of the resonance areas of methylene protons (B and $D$ in Figure 1), and the values in parentheses were determined from methyl and methylene protons (A and D in Figure 1).

d $M_{n}=920$ (by VPO).

and high EMA contents $\left(\eta_{\mathrm{sp}} / c, c a .0 .3\right.$; [EMA]/ [EO], $c a .10)$ were insoluble in methanol, while the copolymers having low molecular weights and high PEO contents $\left(\eta_{\mathrm{sp}} / c<c a .0 .1\right.$; [EMA]/ [EO], ca. 2) were found to be soluble in methanol; the latter copolymers were recovered from filtrates obtained by separation of the insoluble copolymers, and were precipitated by using the solvent-nonsolvent system: methanol-water.

The transesterification of the copolymer (No. 26) with potassium methoxide in $\mathrm{HMPA}\left(70^{\circ} \mathrm{C}\right.$, $100 \mathrm{hr}$ ) gave freed PEO, which was detected by IR spectroscopy. This finding also supports the presence of the grafted chains. However, the quantitative analysis of the grafted ester groups could not be carried out since the transesterification proceeded imperfectly (ca. 30\% based on total ester groups).

\section{DISCUSSION}

It was confirmed that alkali-metal alkoxides derived from PEO could polymerize MMA and EMA, and a copolymer which consisted of polyether- and polymethacrylate-structures could be obtained. This copolymer contained block and graft units. The polymerization with the bifunctional alkoxide derivatives gave both benzene-soluble and -insoluble copolymers. The insoluble (cross-linked) copolymer was considered to be formed by the grafting of the unreacted alkoxide onto the soluble copolymer. It has already been reported by Zilkha, et al., that poly(methyl methacrylate-g-ethylene oxide) could be prepared by transesterification between the pendant ester groups of poly(methyl methacrylate) and the alkoxide derivative of PEO. ${ }^{3}$ In this study, a similar grafting by transesterification was also found to give an insoluble or graft copolymer. This concept can be supported from the fact that the graft copolymer was formed in the polymerization of methacrylates with monofunctional alkoxides derived from PEO.

If the initiation of the polymerization of methacrylates with alkoxides is rapid, the amount of the graft reaction between alkoxides and the pendant ester groups would decrease. However, the amount of the graft reaction was found to increase with an increase in the reactivity of alkoxides: viz., the graft reaction proceeded more readily in the case of the polymerization with potassium alkoxides or the polymerization in the presence of large amounts of HMPA. This fact shows that selectivity in the reaction of methacrylates with alkoxides decreases with an increase in the reactivity of alkoxides, and that the reaction of alkoxides with the ester groups occurs in preference to the reaction with the carbon-carbon double bond in methacrylates. In accord with this, it has been reported that the attack of initiators on the ester group of MMA and/or the metallation of MMA occurred during the polymerization of MMA with highly reactive initiators such as $n$-butyllithium, ${ }^{8}$ and no polymer was produced through the attempted 
polymerization carried out at ambient temperature. ${ }^{9}$

In the polymerization with monofunctional alkoxides no cross-linked polymer was formed, as had been expected, but the graft reaction was found to proceed. Unfortunately, in the present stage a "pure" block copolymer was not obtained by our polymerizations with alkoxides derived from PEO. This polymerization method, however. seems to be of greater advantage than Zilkha's method $^{3}$ for producing graft copolymers composed of PEO and polymethacrylates, because in our method methacrylate monomers can be used as the starting material.

\section{REFERENCES}

1. M. Tomoi, K. Sekiya, and H. Kakiuchi, Polymer J., 6, 438 (1974).

2. (a) R. J. Ceresa, "Block and Graft Copolymers," Encyclopedia of Polymer Science and Technol- ogy, Vol. 2, Interscience Publishers, Inc., New York, N.Y., 1965, p 485; (b) Y. Ikada, "Molecular Design in Polymer Science (Japanese)" Vol. 1, The Society of Polymer Science, Japan, Ed., Baifukan, Tokyo, 1972, p 131.

3. M. A. Twaik, M. Tahan, and A. Zilkha, J. Polym. Sci., Part A-1, 7, 2469 (1969).

4. (a) A. V. Tobolsky and A. Rembraum, J. Appl. Polym. Sci., 8, 307 (1964); (b) Y. Minoura, T. Kasuya, S. Kawamura, and A. Nakano, $J$. Polym. Sci., Part A-1, 5, 43 (1967); (c) J. Galin, M. Galin, and P. Calme, Makromol. Chem., 134, 273 (1970).

5. J. E. Figueruelo and D. J. Worsfold, Eur. Polym. J., 4, 439 (1968).

6. C. C. Price and D. D. Carmelite, J. Amer. Chem. Soc., 88, 4039 (1966).

7. F. A. Bovey and G. V. D. Tiers, J. Polym. Sci., 44, 173 (1960).

8. N. Kawabata and T. Tsuruta, Makromol. Chem., 86, 231 (1965).

9. L. Lochmann, M. Rodova, and J. Trekoval, $J$. Polym. Sci. Polym. Chem. Ed., 12, 2091 (1974). 